\title{
PLASMONIC NANOSTRUCTURES ARRAY WITH CORRELATED DISORDER FOR AUGMENTED REALITY
}

Herve Bertin ${ }^{l}$, Yoann Brûlé ${ }^{2}$, Giovanni Magno ${ }^{l}$, Thomas Lopez ${ }^{l}$, Philippe Gogol ${ }^{l}$, Boris Gralak ${ }^{2}$, David Barat ${ }^{3}$, Guillaume Demésy ${ }^{2}$, Beatrice Dagens ${ }^{1}$

${ }^{I}$ Centre de Nanosciences et de Nanotechnologies, CNRS, Université Paris-Sud, Université Paris-Saclay, Boulevard Thomas Gobert, 91120 Palaiseau, France

${ }^{2}$ Aix Marseille Univ, CNRS, Centrale Marseille, Institut Fresnel, Marseille, France

${ }^{3}$ PSA AUTOMOBILES S.A., Direction Scientifique, Centre technique de Vélizy, route de Gisy, Vélizy-Villacoublay, F-78140, France

Augmented reality makes life easier for users in different domains like education, industry, and medicine. Head-up displays (HUD), used in the automotive domain, can be seen as one early development of augmented reality systems. HUD consists in reflecting a colored display on a transparent glass plate which indicates driving relevant information to the driver. In order to reduce display consumption and/or to obtain sufficient contrast in case of sunny weather, it is necessary to increase the plate reflectance at image color wavelengths. More precisely, the challenge is to develop a plate with simultaneously high reflectance, high angular tolerance and high transparency, providing a clear reflected picture and a clear view of the landscape behind the plate. Among different technologies, localized plasmonics, based on noble metal nanoparticles (NPs) resonances, enables local modification of colors and versatile HUD plate designs [1,2]. Usually, designs of resonant NPs arrays are performed considering periodic layout, more suitable for current numerical tools. However, disordered arrangement presents several advantages: attenuated light diffraction, possible low cost and large scale fabrication, versatile design...

(a)

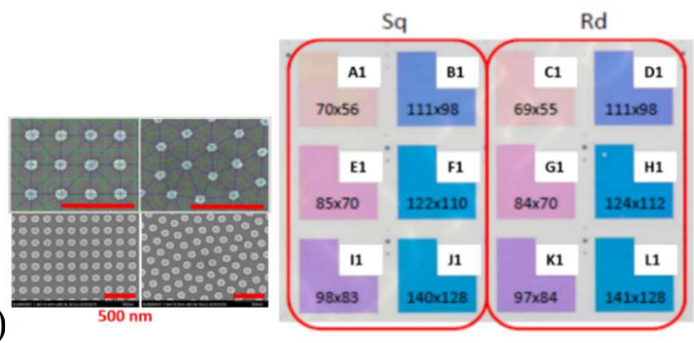

(c)

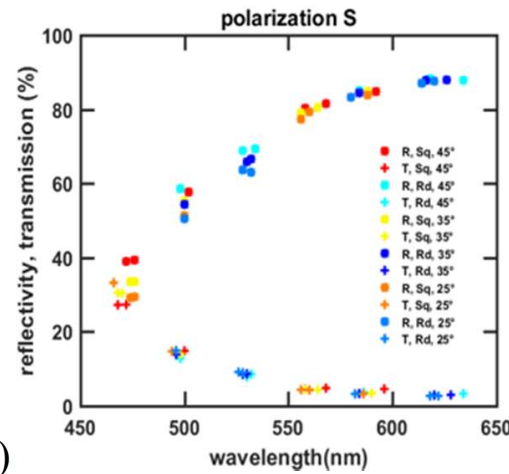

(b)
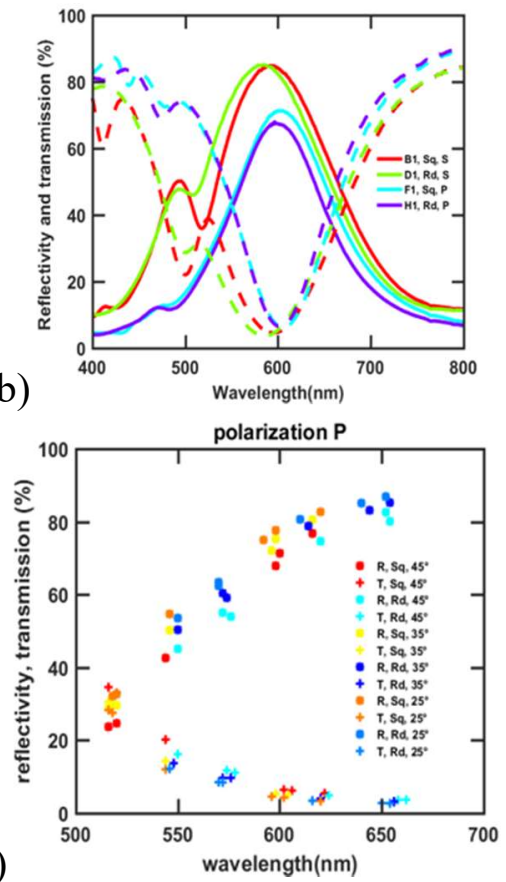

Fig. 1. Fabricated samples: (a) SEM images of the square lattice (left) and correlated disorder (right) NPs arrangements. Backlit photography of the sample with pairs of "Sq" and "Rd" arrays with 200nm (Sq) and $216 \mathrm{~nm}(\mathrm{Rd})$ average interparticle distances and different ellipses diameters (formatted as "a x b" given in $\mathrm{nm}$ ). (b) Reflectance and transmittance spectra for Sq and Rd arrays with identical NPs and both polarizations. $(\mathrm{c}, \mathrm{d})$ Reflectance and transmittance peaks spectral positions of all arrays and different angles for both polarizations. 
Here we first experimentally show that subwavelength period square lattice NPs arrays present similar reflection and transmission properties to the corresponding disordered ones with the same spatial density, regardless of the resonance wavelength, the polarization ( $\mathrm{p}$ or $\mathrm{s}$ ) and the angle of the incident light (Fig. 1). The resonance peaks are tuned by modifying the size of the NPs. The reflectance maxima follow the plasmonic resonance intensity, dependent on the wavelength. The targeted color in this work is amber, corresponding to $590 \mathrm{~nm}$ wavelength. NPs resonant at $590 \mathrm{~nm}$ are considered in the following.

Then, by decreasing the NPs spatial density to increase the plate transparency, possible diffraction orders and surface lattice resonances [3] of the square lattice reflectance spectrum disappear in the case of the disordered array (Fig. 2 a,b). Finally, a wide range of reflectance/transmittance ratio can be obtained by using NPs disordered spatial arrangement, simultaneously avoiding diffraction effects in the visible range (Fig. 2c). Experimental and numerical results are in excellent agreement [4].

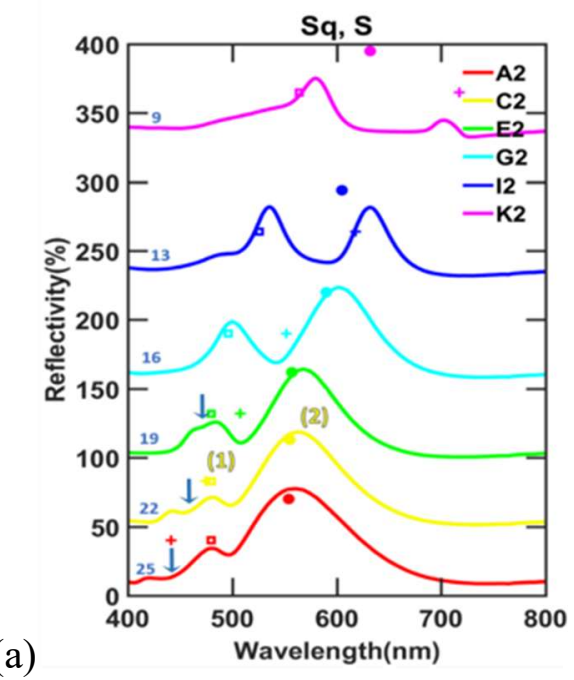

(b)
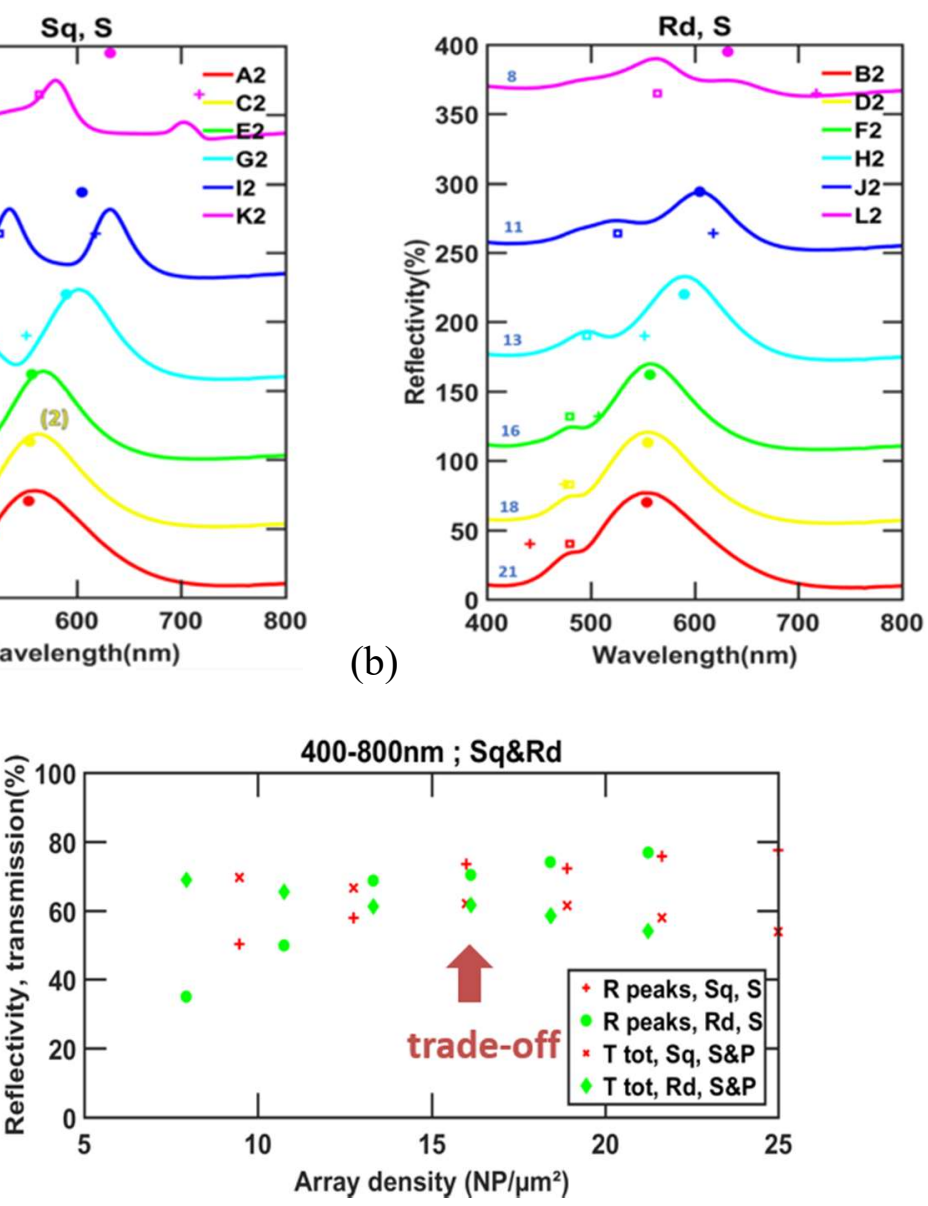

Fig. 2. (a,b) Experimental reflectance spectra for different arrays spatial densities (from 8 to $25 \mathrm{NPs} / \mu \mathrm{m}^{2}$ ) respectively for Sq and Rd arrays. (c) Reflectance maxima in S polarization and averaged transmittance (for both polarizations on the whole visible spectrum) versus the array density. The best compromise is obtained for the density of $16 \mathrm{NPs} / \mu \mathrm{m}^{2}$.

However, whereas the disorder allows suppressing the diffraction rays, it also may induce light diffusion effect. For this reason, we have considered here correlated disorder, in the sense that the relative positions of the NPs is not fully random. The distance between adjacent NPs is constant (by an error bar lower than a few percent): this design is expected to avoid light diffusion effects [5], and also to ease fabrication. 
Two samples of $1 \mathrm{~cm}^{2}$ with disordered arrays of 21 and $16 \mathrm{NPs} / \mu \mathrm{m}^{2}$ respectively have been fabricated (Fig. 3) and qualitatively evaluated: no diffusion or diffraction rays appear.
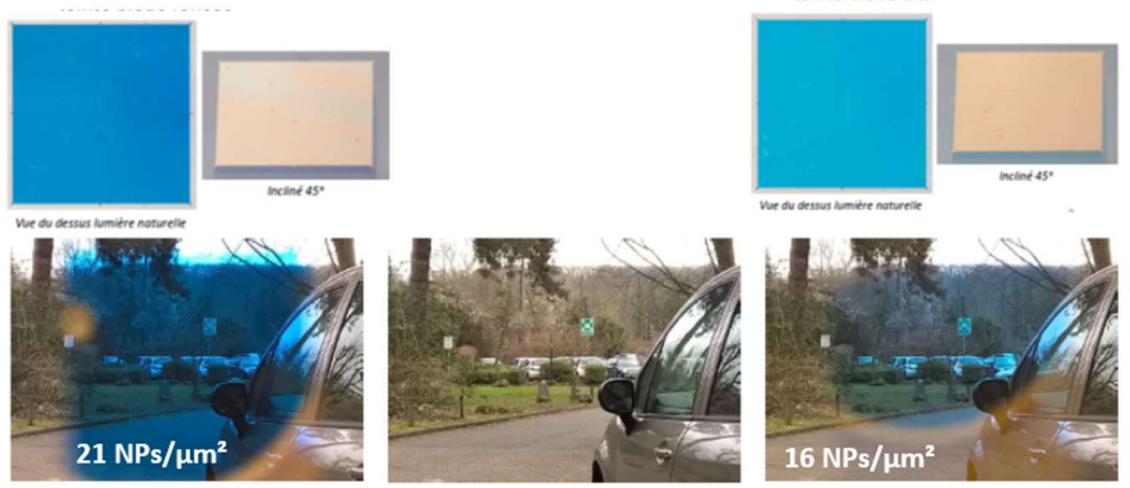

Fig. 3. From left to right, down: qualitative observation of a landscape through a plate of respectively 21 $\mathrm{NPs} / \mu \mathrm{m}^{2}$, null and $16 \mathrm{NPs} / \mu \mathrm{m}^{2}$ array spatial density. Up: photographs of the backlit and illuminated corresponding plates.

Then the sample with the lowest density, corresponding to the best reflectance/transmittance compromise, has been tested in a miniaturized lab HUD bench (Fig. 4). There, the objective is to evaluate the relevance of the design in a realistic situation. Clearly, the reflectance at amber color is significantly increased with respect to uncoated glass, without degrading the quality of the landscape view through the sample.


Fig. 4. (a) Road image without (left) and through (middle) the plasmonic plate. (right) HUD like experiment with the fabricated plasmonic plate, and (b) color description on color gamut (clear blue square), to be compared with reflected image on glass only (dark blue square), perfect mirror (white circle) and with '590 nm' color (black square). (c) Corresponding reflectance at $590 \mathrm{~nm}$.

To conclude, these promising results pave the way for HUD systems and could find some innovative applications in the transport industry such as in the automotive or aeronautic sectors for displays, human-machine interfaces, and sensors. 
This work has been supported by the ANR funded project PLANISSIMO (ANR-12-NANO-0003) and by the french RENATECH network.

[1] Barnes, W.L., Dereux, A \& Ebbesen, T. W. Surface plasmon subwavelength optics. Nature 424, 824-830 (2003).

[2] Y. Brûlé, G. Demésy, A.-L. Fehrembach, B. Gralak, E. Popov, G. Tayeb, M. Grangier, D. Barat, P. Gogol, and B. Dagens, "Design of metallic nanoparticles gratings for filtering properties in the visible spectrum," Appl. Opt. 54,010359 (2015)

[3] R. Guo, T. K. Hakala, and P. Törmä, "Geometry dependence of surface lattice resonances in plasmonic nanoparticle arrays," PHYSICAL REVIEW B 95, 155423 (2017)

[4] H. Bertin, Y. Brûlé, G. Magno, T. Lopez, P. Gogol, L. Pradere, B. Gralak, D. Barat, G. Demésy, and B. Dagens, "Correlated disordered plasmonic nanostructures arrays for augmented reality," ACS Photonics, 2018, 5 (7), pp 2661-2668.

[5] Conley, G.M.; Burresi, M.; Pratesi, F.; Vynck, K.; Wiersma, D.S. Light Transport and Localization in TwoDimensional Correlated Disorder. Phys. Rev. Lett. 2014, 112, 143901. 